\title{
Functional analysis of COP1 and SPA orthologs from Physcomitrella and rice during photomorphogenesis of transgenic Arabidopsis reveals distinct evolutionary conservation
}

\author{
Aashish Ranjan ${ }^{1,3}$, Stephen Dickopf ${ }^{1}$, Kristian K Ullrich², Stefan A Rensing ${ }^{2}$ and Ute Hoecker ${ }^{1^{*}}$
}

\begin{abstract}
Background: Plants have evolved light sensing mechanisms to optimally adapt their growth and development to the ambient light environment. The COP1/SPA complex is a key negative regulator of light signaling in the well-studied dicot Arabidopsis thaliana. COP1 and members of the four SPA proteins are part of an E3 ubiquitin ligase that acts in darkness to ubiquitinate several transcription factors involved in light responses, thereby targeting them for degradation by the proteasome. While COP1 is also found in humans, SPA proteins appear specific to plants. Here, we have functionally addressed evolutionary conservation of COP1 and SPA orthologs from the moss Physcomitrella, the monocot rice and the dicot Arabidopsis.
\end{abstract}

Results: To this end, we analyzed the activities of COP1- and SPA-like proteins from Physcomitrella patens and rice when expressed in Arabidopsis. Expression of rice COP1 and Physcomitrella COP1 protein sequences predominantly complemented all phenotypic aspects of the viable, hypomorphic cop 1-4 mutant and the null, seedling-lethal cop 1-5 mutant of Arabidopsis: rice COP1 fully rescued the constitutive-photomorphogenesis phenotype in darkness and the leaf expansion defect of cop 1 mutants, while it partially restored normal photoperiodic flowering in cop 1. Physcomitrella COP1 partially restored normal seedling growth and flowering time, while it fully restored normal leaf expansion in the cop 1 mutants. In contrast, expression of a SPA ortholog from Physcomitrella (PpSPAb) in Arabidopsis spa mutants did not rescue any facet of the spa mutant phenotype, suggesting that the PpSPAb protein is not functionally conserved or that the Arabidopsis function evolved after the split of mosses and seed plants. The SPA1 ortholog from rice (OsSPA1) rescued the spa mutant phenotype in dark-grown seedlings, but did not complement any spa mutant phenotype in light-grown seedlings or in adult plants.

Conclusion: Our results show that COP1 protein sequences from Physcomitrella, rice and Arabidopsis have been functionally conserved during evolution, while the SPA proteins showed considerable functional divergence. This may - at least in part - reflect the fact that COP1 is a single copy gene in seed plants, while SPA proteins are encoded by a small gene family of two to four members with possibly sub- or neofunctionalized tasks.

Keywords: Photomorphogenesis, Light signal transduction, Flowering time, COP1, SPA1, Evolution, Physcomitrella, Rice, Arabidopsis

\footnotetext{
* Correspondence: hoeckeru@uni-koeln.de

'Botanical Institute and Cluster of Excellence on Plant Sciences (CEPLAS),

Biocenter, University of Cologne, Zülpicher Str. 47b, 50674 Cologne, Germany

Full list of author information is available at the end of the article
} 


\section{Background}

Since plants use sunlight as their primary source of energy they have evolved mechanisms of light sensing in order to optimally adjust their growth and development accordingly. Light-adapted responses are particularly obvious during seedling growth. Dark-grown seedlings usually exist under soil cover and therefore respond with etiolation, showing a long hypocotyl, small and closed cotyledons, an apical hook and a lack of chlorophyll synthesis. Light-grown seedlings, in contrast, are green and exhibit a short hypocotyl, open, expanded and green cotyledons and no apical hook. Other light-induced responses include phototropism, leaf expansion, the shade avoidance response and photoperiodic flowering $[1,2]$. To sense the light, plants have several classes of photoreceptors: the red (R) and far-red (FR) sensing phytochromes, the blue (B)/UV-A responsive cryptochromes, phototropins and ZEITLUPE family members and the recently identified UV-B sensing UV-RESISTANCE LOCUS 8 (UVR8) protein [3-6].

The molecular events during light signal transduction are best understood in the model species Arabidopsis. After activation by light, phytochrome and cryptochrome photoreceptors inhibit the activity of a key negative regulator of light signal transduction, the CULLIN4 (CUL4)-dependent E3 ubiquitin ligase complex CONSTITUTIVELY PHOTOMORPHOGENIC1/SUPPRESSOR OF PHYA-105 (COP1/ SPA). In darkness, COP1/SPA acts to ubiquitinate activators of the light response, such as the transcription factors ELONGATED HYPOCOTYL5 (HY5), LONG HYPOCOTYL IN FR 1 (HFR1), B-BOX DOMAIN PROTEINS (BBX) proteins, PRODUCTION OF ANTHOCYANIN PIGMENT1 (PAP1) and PAP2 as well as several photoreceptors, thereby targeting them for degradation in the proteasome. In light-grown plants, in contrast, COP1/SPA activity is suppressed and the target proteins can accumulate and mediate light-regulated gene expression and photomorphogenesis [7-11]. Hence, mutants defective in $C O P 1$ or in all four members of the SPA gene family show constitutive photomorphogenesis, exhibiting features of light-grown seedlings in complete darkness [12,13]. Besides controling seedling growth in response to light, the COP1/SPA complex is involved in multiple other lightinduced responses, such as anthocyanin biosynthesis, leaf expansion, shade avoidance responses and photoperiodic flowering $[7,11,14-19]$. COP1/SPA also acts downstream of the UV-B receptor UVR8, but in contrast to $R$ and $B$ signaling - where COP1 acts as a repressor of light signaling - COP1/SPA functions as a positive regulator of the UV-B response [20].

The COP1/SPA complex likely forms a tetramer with two COP1 and two SPA proteins. COP1 and SPA proteins interact with each other via their respective coiled-coil domains [21-24]. COP1 and the four SPA proteins (SPA1-
SPA4) share further structural similarity in that they contain related C-terminal WD-repeat domains which have dual roles in substrate recruitment and binding of DAMAGED DNA-BINDING PROTEIN1 (DDB1) of the CUL4 complex [11]. In their N-termini, COP1 and SPA proteins have distinct sequences, with COP1 containing a RING finger domain and SPA proteins carrying a kinase-like domain $[25,26]$. The mechanisms involved in light-mediated inhibition of COP1/SPA activity are not well understood but likely involve light-induced interaction of cryptochromes with SPA1, light-induced degradation of SPA1 and SPA2 as well as light-mediated nuclear exclusion of COP1 [27-33].

The four SPA proteins share highest sequence similarity to each other in their WD-repeat domain. Sequence conservation of the $\mathrm{N}$-terminal domain is relatively low and mostly limited to the kinase-like domain. Based on sequence similarity, the four SPA proteins fall into two subgroups with SPA1 and SPA2 forming one subgroup and SPA3 and SPA4 forming the other subgroup [13]. Genetic analysis of spa mutants indicated that the four SPA genes have partly redundant but also distinct functions in plant growth and development $[13,27,34]$.

COP1 functions have also been described in other flowering plant species. In rice, the COP1 ortholog PETER PAN SYNDROME1 (PPS) shortens the juvenile phase, a phenotype not reported for Arabidopsis, and delays flowering in short and long day [35]. The COP1 ortholog of pea, LIGHT-INDEPENDENT PHOTOMORHOGENESIS1 (LIP1), regulates seedling growth by affecting gibberellic acid levels [36,37]. In apple, $M d C O P 1$ affects anthocyanin levels in the fruit peel [9]. COP1 also exists in non-plant lineages, e.g. humans, where hCOP1 acts as an E3 ubiquitin ligase to control the protein stability of a number of transcription factors, e.g. p53 or cJun [38]. SPA genes, in contrast, appear to be specific to plants, which indicates that human COP1 functions without a need for SPA proteins. This suggests that $S P A$ genes might have evolved to place COP1 activity under the control of light. Indeed, the $\mathrm{N}$-terminus of SPA1 was shown to be involved in the blue-light dependent interaction of SPA1 with cryptochrome photoreceptors [31,32].

Whole genome sequencing has shown that COP1 and $S P A$ genes exist in early diverged land plants, such as in the moss Physcomitrella patens. There are a number of light responses known in Physcomitrella, such as chloroplast movement, phototropism, caulonema branching and gametophore growth [39] as well as UV-B responses akin to those in Arabidopsis [40]. While COP1 is a single copy gene in rice and Arabidopsis [11], genome sequence information predicted a total of nine paralogs in $P$. patens $[41,42]$. Both the rice and Physcomitrella genomes contain two SPA-related genes each [41-43]. Physcomitrella has functional phytochrome and cryptochrome photoreceptors 
[39,44-47], allowing the possibility that PpCOP1 and PpSPA genes may also function in light signal transduction in Physcomitrella.

To address the evolutionary conservation of COP1 and SPA protein sequences, we expressed COP1 and SPA coding sequences from rice and Physcomitrella in the respective cop 1 and spa mutant backgrounds of Arabidopsis. Our results show that COP1 sequences are functionally much more conserved than SPA sequences, suggesting that gene duplication of $S P A$ genes in the flowering plant lineage has contributed to divergence of SPA gene functions.

\section{Results}

\section{A comparison of Physcomitrella, rice and Arabidopsis} COP1 and SPA protein sequences

Based on the v1.6 genome annotation currently available [48], the Physcomitrella genome contains 9 COP1like genes (Figure 1; Additional file 1: Figure S1), as was predicted previously based on v1.2 [41]. The predicted PpCOP1 protein sequences share $61-82 \%$ amino acid sequence identity among each other and 55-64\% amino acid sequence identity with the Arabidopsis COP1 protein. The COP1 ortholog from rice (PPS [35], here for clarity from now on referred to as OsCOP1) and Arabidopsis COP1 share approx. 70\% identical amino acids. Like Arabidopsis COP1, all predicted PpCOP1 proteins and OsCOP1 contain a RING finger motif, at least one coiled-coil domain and a WD40 repeat domain (Figure 1; Additional file 1: Figure S1C; Additional file 2: Figure S2, Additional file 3: Figure S3).

While the COP1 gene family has expanded in Physcomitrella as compared to a single COP1 gene reported in flowering plant species, there are only two predicted $S P A$ genes in Physcomitrella. These two PpSPA genes are very similar to each other $(89 \%$ amino acid identity of the predicted proteins), suggesting that they represent recent duplication events based on an ortholog of AtSPA1/2 (Figure 1; Additional file 1: Figure S1A, B; Additional file 4: Figure S4). We named the two Physcomitrella SPA genes PpSPAa (Pp1s59_66V6.1) and PpSPAb (Pp1s30_295V6.1). There are two predicted rice SPA proteins of which each groups with one subclass from Arabidopsis (AtSPA1/2, AtSPA3/4) (Figure 1; Additional file 1: Figure S1A, B), evidencing that two paralogs were already present in the last common ancestor of monocots and dicots. The SPA1/SPA2-like rice SPA was more similar to Arabidopsis SPA1 than to Arabidopsis SPA2. We therefore refer to this rice SPA as rice SPA1-like or OsSPA1 (Os05g49590.1). The predicted SPA3/SPA4-like SPA from rice equally resembles Arabidopsis SPA3 and SPA4 protein sequences. We therefore refer to it as rice SPA3/4-like or OsSPA3/4 (Os01g52640.1). The predicted domain structures of Physcomitrella and rice SPA proteins are similar to those from Arabidopsis SPA proteins: they all contain an N-terminal kinase-like domain, a coiled-coil domain and seven WD40-repeats (Figure 1; Additional file 1: Figure S1C, Additional file 3: Figure S3, Additional file 4: Figure S4). Similar to Arabidopsis SPA proteins, the kinase-like domains from rice and Physcomitrella SPA proteins share only limited sequence conservation with bona fide Ser/Thr kinase consensus motifs because amino acid residues that are normally highly conserved in Ser/Thr kinases are not conserved in PpSPA and OsSPA proteins. Nevertheless, sequences in the kinaselike domain that are conserved among the four Arabidopsis SPA proteins are also highly conserved in OsSPA and PpSPA proteins (Additional file 4: Figure S4). All SPA sequences in Arabidopsis, rice and Physcomitrella contain a predicted coiled-coil domain (Additional file 3: Figure S3), though the sequence of the respective coiled-coil domain is not strongly conserved among Arabidopsis, rice or Physcomitrella SPA proteins. This suggests a structural rather than sequence-based conservation of this domain in the SPA proteins. The SPA protein sequences are most conserved within the WD40-repeat domain, with Physcomitrella SPAa and SPAb showing $65 \%$ amino acid identity with AtSPA1 compared with $42 \%$ when aligning the complete protein sequences.

Rice and Physcomitrella also contain predicted orthologs of the Arabidopsis RUP genes. Arabidopsis RUP proteins consist of COP1/SPA-like WD40 repeats and function as negative regulators of UV-B signaling $[49,50]$. The rice genome contains 1 ortholog of RUP, while Physcomitrella has two predicted RUPs (Figure 1; Additional file 1: Figure S1, Additional file 5: Figure S5).

\section{Functional analysis of COP1-like proteins from rice and Physcomitrella in the hypomorphic cop 1-4 mutant of Arabidopsis}

In order to address the evolutionary conservation of COP1 and SPA function, we expressed the coding sequence of Physcomitrella, rice and - as a control - Arabidopsis COP1 and SPA genes in transgenic Arabidopsis cop1 and spa mutants, respectively, to subsequently evaluate whether the transgenes complement the respective mutant phenotypes. Though protein detection in the transgenic plants is desirable, we did not add an epitope tag to the coding sequence because a tag might negatively affect protein function. Among the nine $P p C O P 1$ genes, we chose the one with the highest sequence similarity to AtCOP1, based on BLAST scores, for the complementation study (Pp1s135_17V6.1, PpCOP1a, Figure 1). The coding sequences of OsCOP1, PpCOP1a and AtCOP1 were placed under the control of the $35 S$ constitutive promoter and introduced into the hypomorphic cop 1-4 mutant and into the cop1-5 null mutant of Arabidopsis. While the cop1 null mutant is seedling lethal, the cop1- 


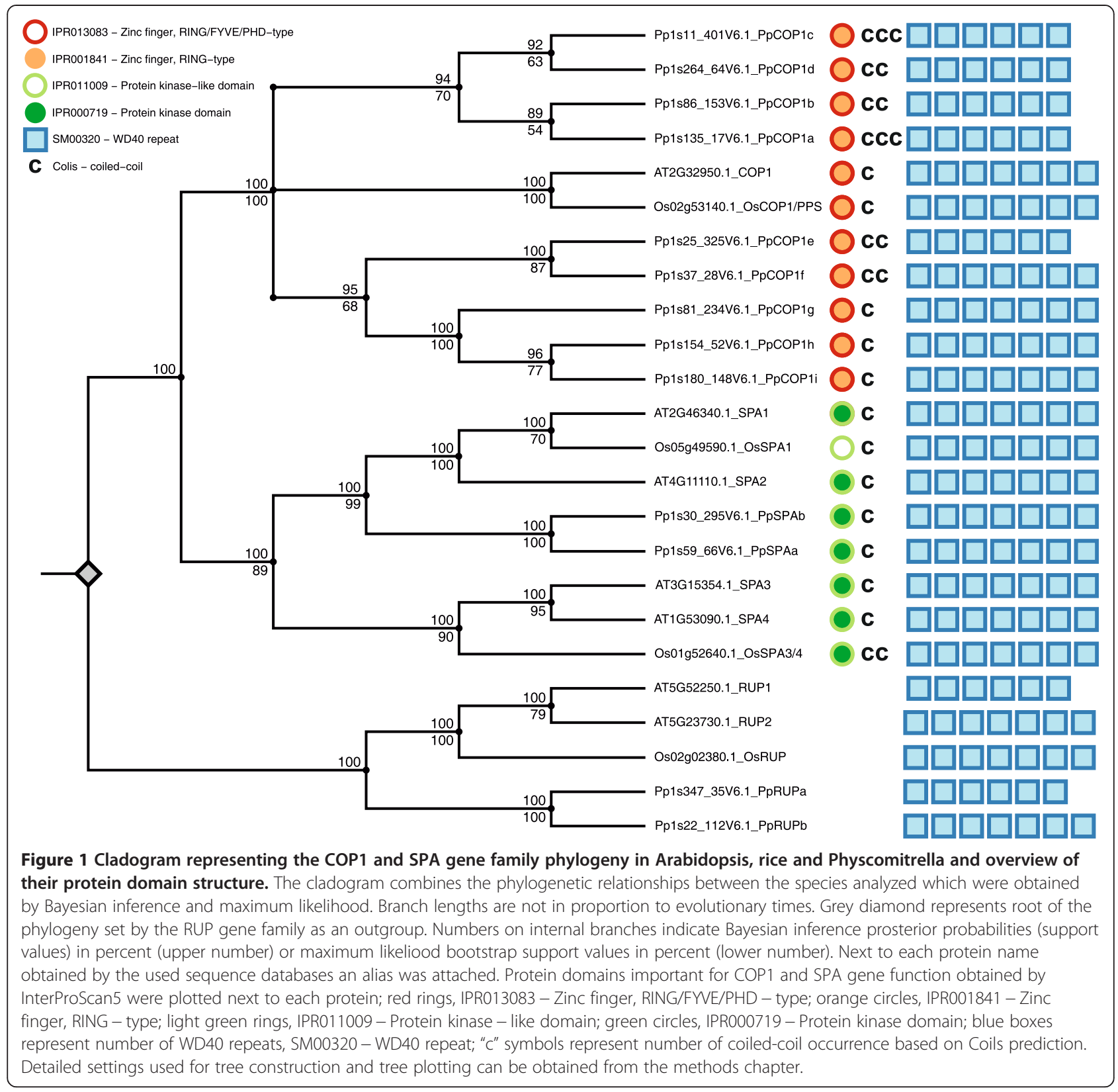

4 mutant is viable, producing a truncated COP1 protein lacking the C-terminal WD-repeat domain [12,51].

cop1-4 mutant seedlings undergo constitutive photomorphogenesis in darkness, exhibiting short hypocotyls and open cotyledons (Figure 2A [51]). Transgenic cop1-4 seedlings expressing the Arabidopsis COP1 gene or rice COP1 ortholog fully etiolated in darkness and thus resembled the wild type. Hence, AtCOP1 and OsCOP1 fully complemented the cop1-4 mutant phenotype in darkness. Transgenic cop 1-4 seedlings carrying the P $p C O P 1 a$ transgene showed a partial rescue of the cop1-4 mutant phenotype in darkness: PpCOP1a lines exhibited a longer hypocotyl than cop1-4 in darkness but failed to fully etiolate, as indicated by the open cotyledons and the lack of an apical hook (Figure 2A). Of 25 independent $\mathrm{PpCOP1a}$ lines investigated, none showed a full rescue of the cop 1-4 mutant phenotype in darkness. When grown in light of low to intermediate fluence rates, cop1-4 mutant seedlings exhibited a shorter hypocotyl than the wild type ([51], Figure 2B). This mutant phenotype was similarly complemented by all three transgenes, AtCOP1, OsCOP1 and $\mathrm{PpCOP} 1 \mathrm{a}$ (Figure 2B).

Besides the constitutive photomorphogenesis in seedlings, cop 1-4 mutants exhibit mutant phenotypes in the adult plant: cop 1-4 mutant plants are small and dwarfed and they flower earlier than the wild type, particularly 


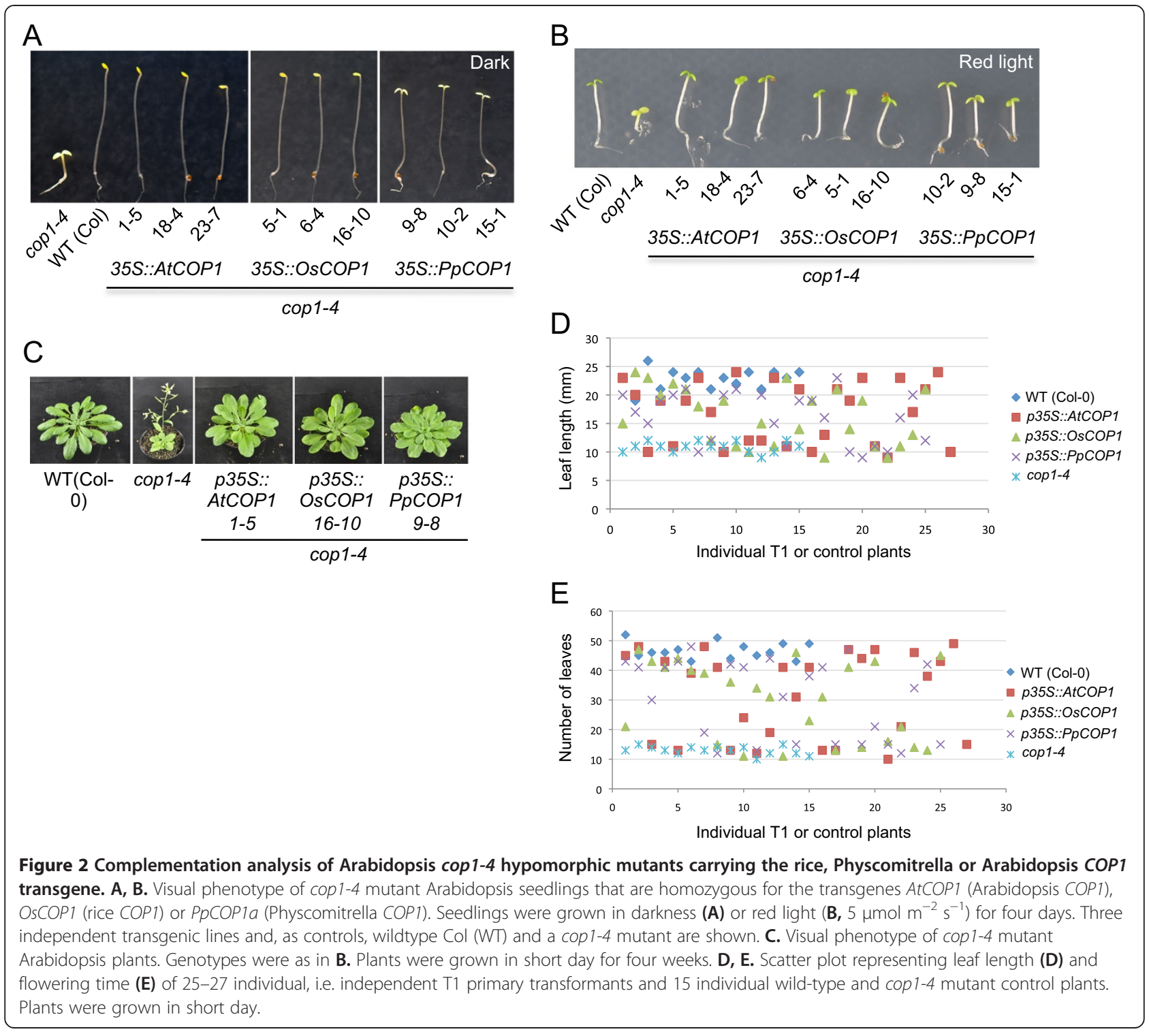

under short day conditions [51]. Transgenic AtCOP1, OsCOP1 and PpCOP1a cop1-4 mutant lines were similar in size as the wild type and flowered at a similar time as the wild type (Figure 2C,D,E). For each of the three transgenes, about half of the transgenic T1 plants showed full rescue of the cop1-4 mutant adult phenotypes (Figure 2D, E). Hence, $O s C O P 1$ and $P p C O P 1 a$, like $A t C O P 1$, were able to fully complement the cop1-4 mutant phenotypes in adult plants.

Functional analysis of COP1-like proteins from rice and Physcomitrella in the cop1-5 null mutant of Arabidopsis Since the cop1-4 mutant allele expresses a truncated COP1 protein retaining the N-terminal part of COP1 including the coiled-coil domain [51], rescue of the cop1-4 mutant phenotype by expression of OsCOP1 or PpCOP1a might depend on the presence of the truncated COP1-4 protein, especially since the retained coiled-coil domain might allow protein-protein interaction with OsCOP1 and PpCOP1a. We therefore introduced the transgenes also into the cop1-5 null mutant background by transforming cop 1-5/+plants and by crossing transgenic cop1-4 mutants with cop1-5/+plants. Homozygous cop1-5 (-/-) mutant seeds in the progeny could be easily recognized by their black seed color, though they mostly failed to germinate [51]. Assuming Mendelian segregation of the seedlinglethal cop1-5 mutant phenotype, the penotypic effect of the transgenes should be analyzable in the respective T2 generations based on the segregation ratio of mutant and wild-type phenotypes. However, we found a much reduced transmission frequency of the cop1-5 mutant allele when compared to the COP1 wild-type allele, thus making the 
analysis of segregating populations ambiguous. We therefore generated homozygous cop1-5 mutant lines that were also homozygous for the respective transgene. Figure 3A shows that AtCOP1 and OsCOP1 fully restored a wildtype phenotype in dark-grown homozygous cop1-5 mutant seedlings. Hence, the AtCOP1 and OsCOP1 transgenes not only rescued the seedling-lethal phenotype of cop1-5 but also fully complemented its fusca phenotype of constitutive photomorphogenesis and strong anthocyanin production which was described for strong cop1 alleles [51]. PpCOP1a cop1-5 seedlings, in contrast, showed open cotyledons and a slightly shorter hypocotyl than the wild type when grown in darkness (Figure 3A,B). Thus, expression of $P$ COP $1 a$ resulted in partial complementation of the cop1-5 mutant phenotype. In light-grown seedlings, the control construct AtCOP1 fully complemented the cop1-5 mutant phenotype. In contrast, B- and FR-grown OsCOP1 cop1-5 and PpCOP1a cop1-5 seedlings were even taller than wild-type seedlings, especially at higher fluence rates, indicating a reduced response to B and FR when compared to the wild type (Figure 3B; Additional file 6: Figure S6). In $\mathrm{R}$, all transgenic seedlings behaved similar to the wild type (Additional file 6: Figure S6).

Since all three transgenes rescued the seedling-lethal phenotype of cop1-5, we were able to analyze the activity of the transgene also in the adult stage. Transgenic OsCOP1 cop1-5, PpCOP1a cop1-5 and AtCOP1 cop1-5 plants were of similar size as the wild type (Figure $2 \mathrm{C}, \mathrm{D}$ ). With respect to flowering time, transgenic AtCOP1 cop1-5 lines flowered at a similar time as the Ws wild type while transgenic OsCOP1 cop1-5 and, in particular, PpCOP1a cop1-5 lines flowered earlier than the wild type and the AtCOP1 cop1-5 transgenic lines (Figure 2E). These results indicate that the COP1 sequences from rice and Physcomitrella only partially rescued this aspect of the cop1-5 mutant phenotype.

Rice and Physcomitrella SPA protein-coding sequences do not complement the light hypersensitivity-phenotype of the Arabidopsis spa 1 spa3 spa4 triple mutant

To analyze functional conservation of rice and Physcomitrella SPA1-related protein-coding sequences we expressed OsSPA1 and PpSPAb ORFs in an Arabidopsis spa mutant. The two Physcomitrella SPA proteins, SPAa and SPAb are highly similar to each other (89\% amino acid sequence identity) and both share equal sequence similarity to the Arabidopsis SPA1. We therefore chose only one of these SPAs, SPAb, for our analyses. As controls, we included the Arabidopsis SPA1 and SPA4 ORFs because these two SPAs are representative for the partially distinct functions of the four SPA genes [13,15,34]. We transformed these constructs into the spa1 spa3 spa4 triple mutant because this mutant is a viable spa mutant showing defects in multiple phenotypes including seedling deetiolation, leaf expansion and flowering time control [13,15]. Initially, we expressed the SPA coding sequences under the control of the $35 S$ promoter. However, the Arabidopsis 35S::AtSPA1 and 35S:: AtSPA4 constructs produced very low complementation rates $(<10 \%$ of transgenic plants) in the spa triple mutant, an observation we had made before [52]. We therefore proceeded to express the respective SPA coding sequences under the control of the endogenous Arabidopsis AtSPA1 and AtSPA4 5' and 3' regulatory sequences which previously produced very high complementation rates among transgenic spa mutant plants (>90\%) [27,52]. For linguistic simplicity, we will refer to these regulatory sequences as ' promoters' from now on.

spa1 spa3 spa4 triple mutant seedlings etiolate normally in darkness, but have a severely reduced hypocotyl length in weak light when compared to the wild type. Hence, this mutant is strongly hypersensitive to light ([13], Figure 4A). Expression of AtSPA1 from the AtSPA1 promoter fully restored the spa 3 spa4 phenotype in the spa1 spa3 spa4 mutant, thus reflecting the activity of the native SPA1 gene. In contrast, expression of rice OSSPA1 or Physcomitrella PpSPAb from the AtSPA1 promoter did not alter the spa1 spa3 spa4 mutant seedling phenotype in any of the 20 independent transgenic lines analyzed for each construct (Figure 4A). Similarly, when PpSPAb was expressed from the Arabidopsis AtSPA4 promoter, no change in the spa1 spa3 spa4 mutant phenotype was observed, while expression of the control construct AtSPA4::AtSPA4 caused an elongation of the hypocotyl when compared to the spa1 spa3 spa4 progenitor, though the effect of AtSPA4::AtSPA4 was consistently weaker than that of AtSPA1::AtSPA1, as expected [13].

In the adult stage, none of the constructs containing the OsSPA1 or PpSPAb coding sequences complemented the dwarfism or the early flowering time of the spa1 spa3 spa4 mutant (Figure 4B,C,D). Expression of the control constructs AtSPA1::AtSPA1 or AtSPA4::AtSPA4, in contrast, rescued these facets of the spa 1 spa 3 spa4 mutant phenotype to the expected degree $[13,15]$.

To confirm that OsSPA1 and PpSPAb genes are indeed expressed in the transgenic plants, we analyzed SPA transcript levels by semiquantitative RT-PCR. Figure 5 shows that all transgenes were expressed. This indicates that the failure of OsSPA1 and PpSPAb coding sequences to complement the $s p a$ triple mutant phenotype was not caused by a lack of expression of the respective $S P A$ genes.

\section{Functional analysis of SPA orthologs from rice and} Physcomitrella in the constitutively photomorphogenic spa1 spa2 spa3 mutant of Arabidopsis

Since Arabidopsis spa 1 spa 3 spa 4 mutant seedlings analyzed above etiolate normally in darkness, this background precludes a genetic complementation analysis in dark-grown seedlings. We therefore introduced the SPA 


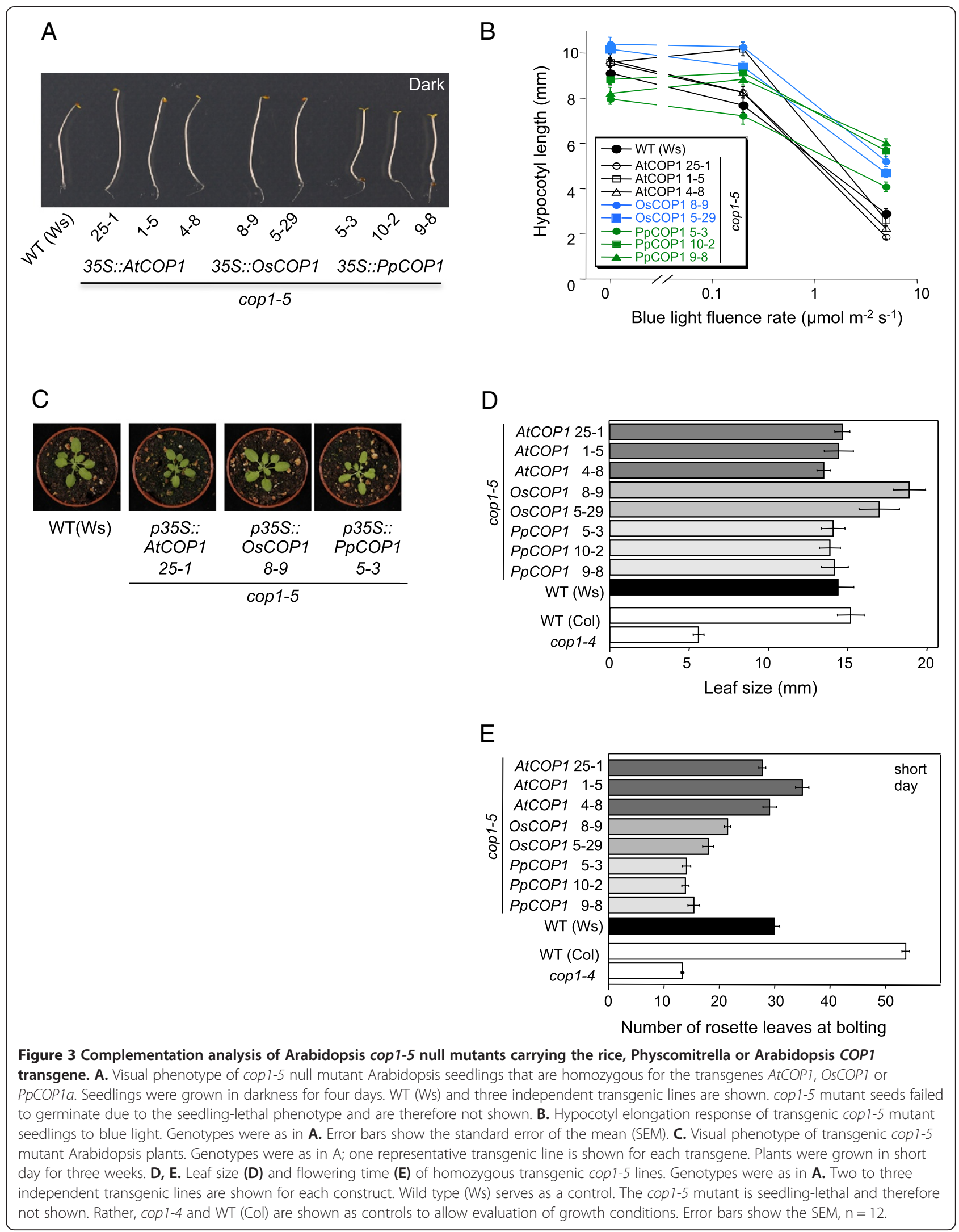




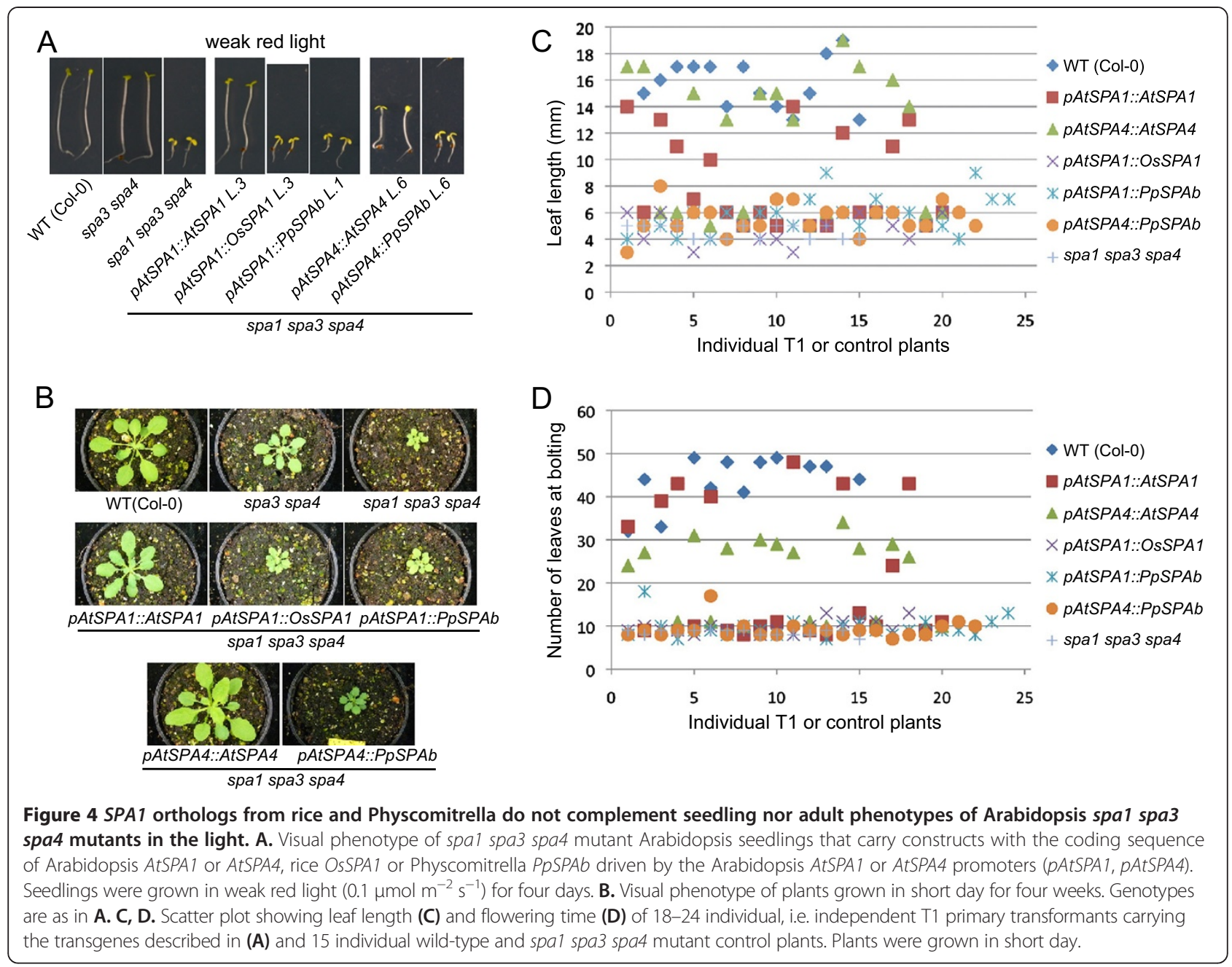

constructs also into the spa1 spa2 spa3 triple mutant which undergoes constitutive seedling photomorphogenesis in darkness (Figure 6), while it develops normally as an adult plant $[13,15]$.

Expression of the control constructs (AtSPA1::AtSPA1; AtSPA4::AtSPA4) fully complemented the spa 1 spa 2 spa 3 mutant phenotype in darkness: all of the AtSPA1::AtSPA1 lines (12/12 independent lines total) and most of the AtSPA4::AtSPA4 lines (10/11 total) exhibited normal skotomorphogenesis in darkness (Figure 6). When expressing the rice SPA1 (AtSPA1::OsSPA1), several transgenic lines showed partial $(8 / 22$ total) or full (1/22 total) complementation of the spa1 spa 2 spa 3 mutant phenotype in darkness (Figure 6). Hence, OsSPA1 appears to be functional in Arabidopsis, though at a much reduced efficiency when compared to AtSPA1. In contrast, none of the 25 transgenic lines expressing Physcomitrella $P p S P A b$ under the AtSPA1 or AtSPA4 promoters showed any rescue of the spa1 spa 2 spa 3 mutant phenotype: these transgenic spa 1 spa3 spa4 seedlings underwent constitutive photomorphogenesis in darkness very similar to the spa1 spa 2 spa 3 mutant progenitor (Figure 6). Hence, PpSPAb was nonfunctional in Arabidopsis. Again, all transgenes were expressed in the respective transgenic lines, as indicated by the presence of the transgene-encoded transcripts (Figure 7).

\section{Discussion}

The COP1/SPA complex of Arabidopsis is a wellcharacterized key negative regulator that actively suppresses the light signaling cascade in dark-grown plants by ubiquitinating transcription factors which mediate the various light responses. The E3 ubiquitin ligase activity is conserved in the mammalian ortholog of COP1 which, however, appears to function without a need for SPA proteins since SPA genes appear to be specific to plants. SPA protein sequences are distinct from COP1 in that they carry a kinase-like domain in the $\mathrm{N}$-terminus $[13,26]$. This kinase-like domain is conserved in Physcomitrella, rice and Arabidopsis SPA proteins and shows a similar divergence in sequence from bona fide Ser/Thr kinase motifs in all three species. This finding suggests on one hand that 

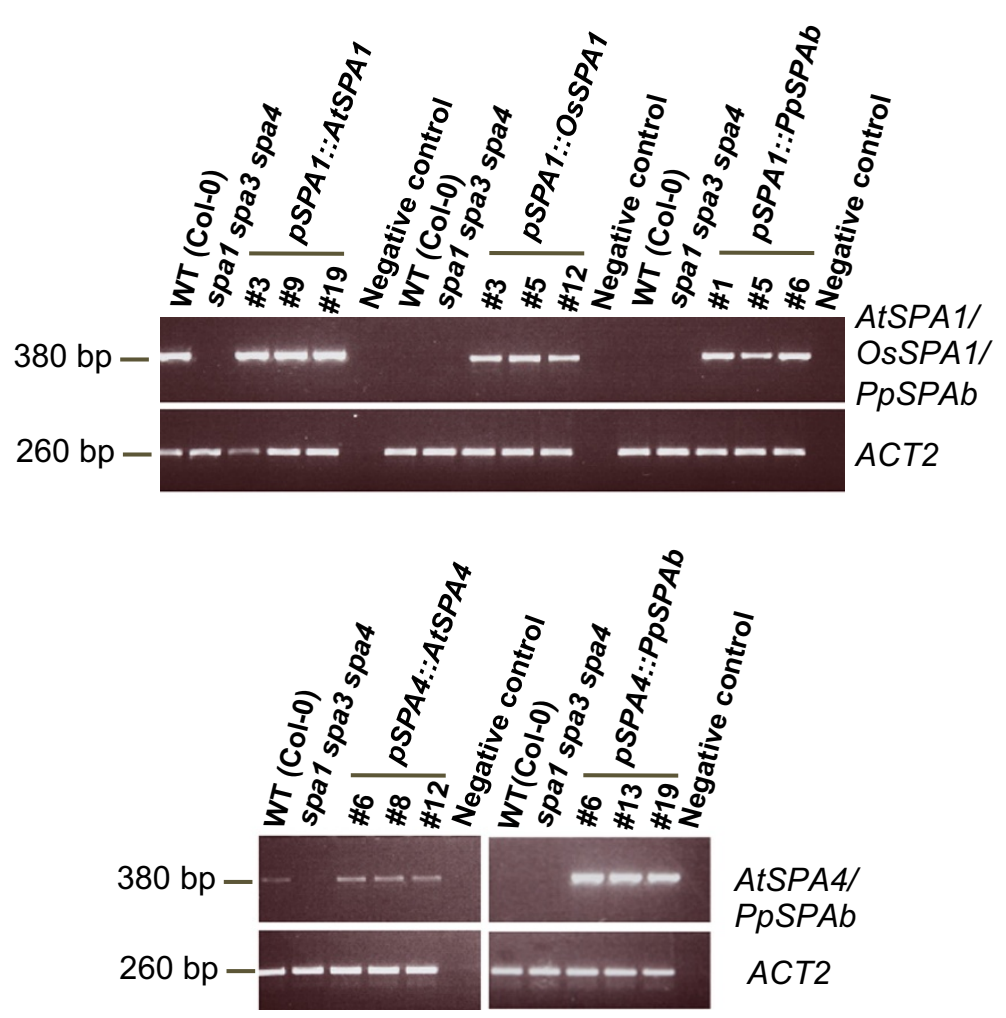

Figure 5 Transcript levels of the transgenes in transgenic spa 1 spa3 spa4 mutant lines. AtSPA1, OsSPA1, PpSPAb and AtSPA4 transcript levels in transgenic seedlings carrying the indicated constructs. Transcript levels were analyzed by semi-quantitative RT-PCR using primers specific for

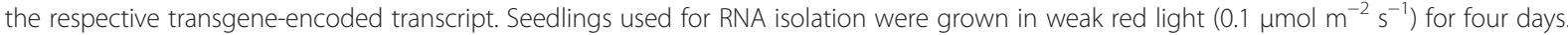
Primers amplifying the ACT2 transcript were used as a control.
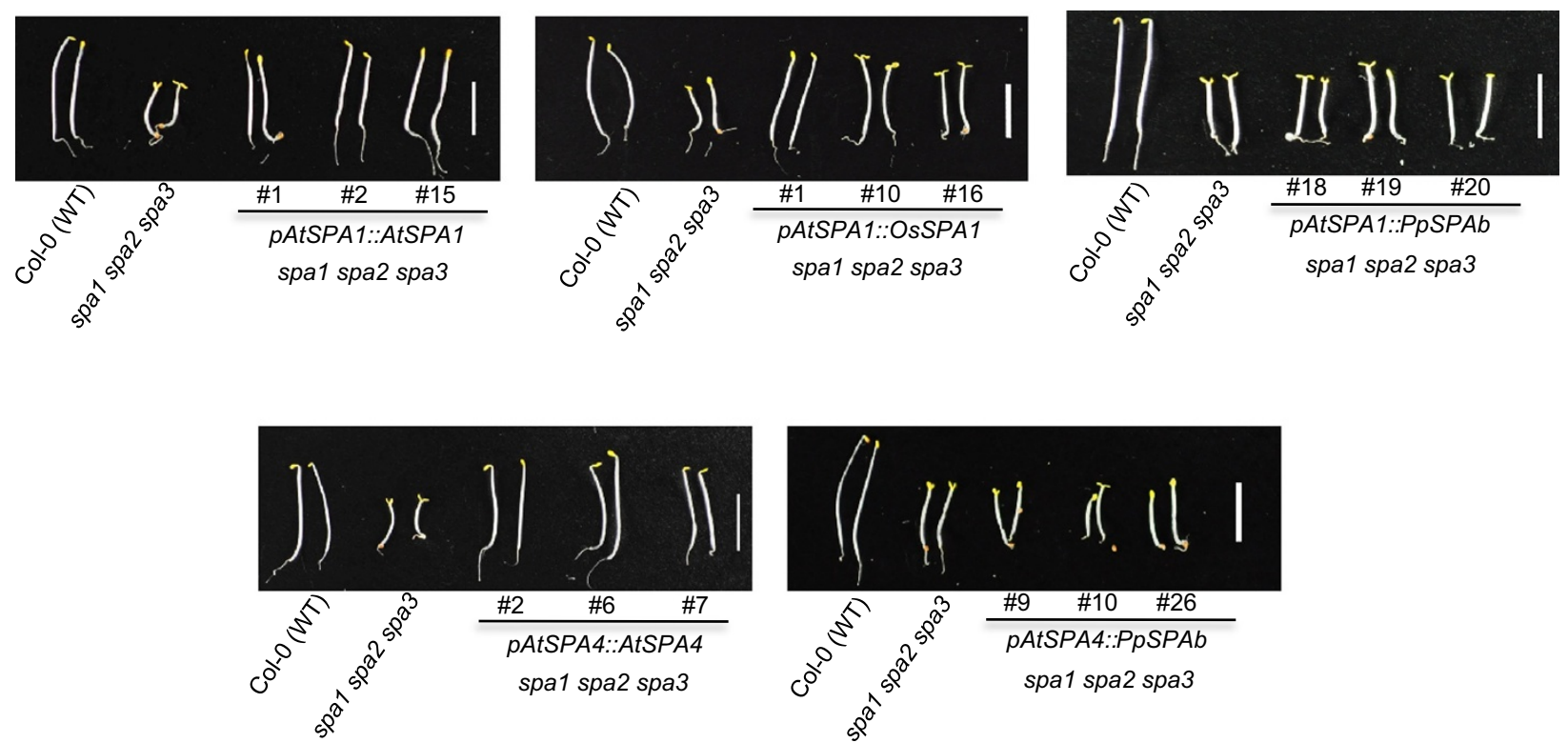

Figure 6 Complementation analysis of dark-grown spa1 spa2 spa3 mutant seedlings carrying rice, Physcomitrella or Arabidopsis SPA1 or SPA1-related transgenes. Visual phenotype of spa1 spa2 spa3 mutant Arabidopsis seedlings that carry constructs with the coding sequence of Arabidopsis AtSPA1, rice OSSPA1, Physcomitrella PPSPAb or Arabidopsis AtSPA4 driven by the Arabidopsis SPA1 or SPA4 promoters (pAtSPA1, pAtSPA4). Seedlings were grown in darkness for four days. 

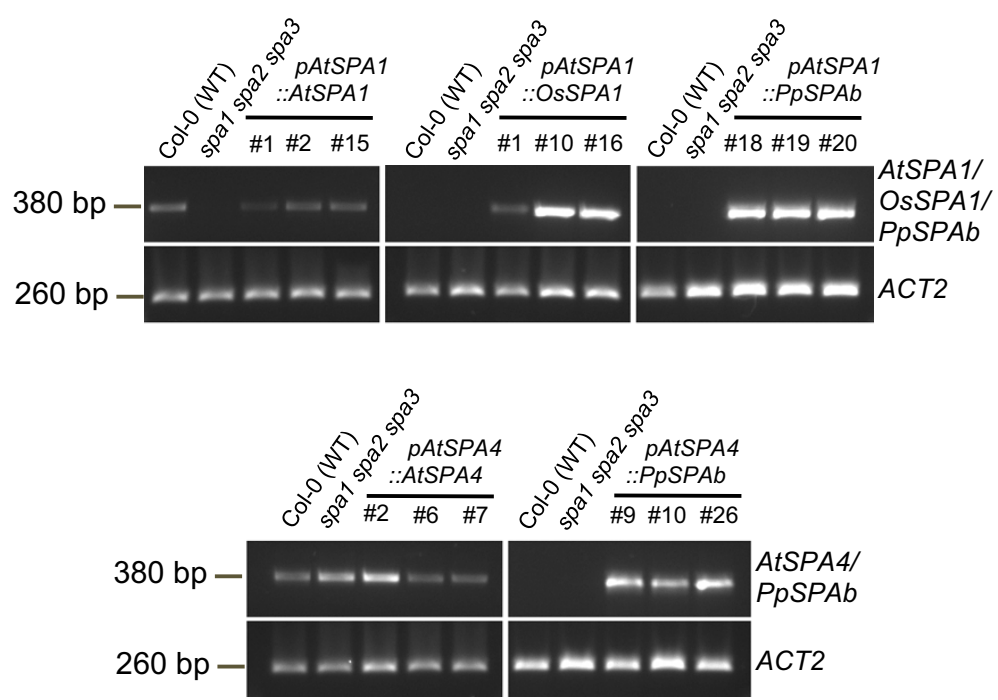

Figure 7 Transcript levels of the transgenes in transgenic spa1 spa2 spa3 mutant lines. AtSPA1, OsSPA1, PpSPAb and AtSPA4 transcript levels in transgenic seedlings carrying the indicated constructs. Transcript levels were analyzed by semi-quantitative RT-PCR using primers specific for the respective transgene-encoded transcript. Seedlings used for RNA isolation were grown in darkness for four days. Primers amplifying the ACT2 transcript were used as a control. Negative controls contained no template DNA.

this kinase-like domain is of functional importance - though its exact role has so far remained elusive [31,32,34,53] - and on the other hand that early in land plant evolution this domain was already divergent in sequence from normal protein kinases.

Our functional analysis clearly shows that PpCOP1a from Physcomitrella is able to mostly replace the functions of COP1 in Arabidopsis. Similarly, rice OsCOP1 was able to mostly complement all aspects of the Arabidopsis cop1 mutant phenotype. These findings suggest that COP1 is under strong negative selection in seed plants. Physcomitrella PpSPAb, in contrast, was incapable of complementing any of the spa mutant phenotypes in transgenic Arabidopsis, strongly suggesting that the PpSPAb protein is non-functional in Arabidopsis. Similarly, expression of the rice OsSPA1 protein in Arabidopsis spa mutants failed to complement any phenotypes of light-grown spa mutant plants and complemented the phenotype of dark-grown seedlings at a much reduced efficiency. These results suggest that SPA-like sequences underwent considerable functional divergence during evolution. However, since we cannot determine the PpSPAb and OsSPA1 protein levels in the transgenic Arabidopsis plants we cannot exclude the possibility that the apparent inactivity of PpSPAb and OsSPA1 in Arabidopsis are due to inefficient translation of the respective mRNAs or due to instability of the respective proteins in Arabidopsis when compared to the native Arabidopsis SPA1 protein. To fully understand the functional conservation between SPA1 from moss, rice and Arabidopsis, it will also be necessary to genetically identify OsSPA1 and PpSPA1 function in rice and Physcomitrella, respectively. Moreover, a protein-protein interaction analysis among the respective COP1 and SPA orthologs will be helpful in analyzing OsSPA1 and PpSPAb activity in Arabidopsis.

We can only speculate why the COP1 gene appears to be subject to much less functional divergence than SPA1. One likely reason is the fact that COP1 is a single-copy gene in flowering plants while SPA proteins are encoded by a small gene family comprising two to four members. Gene duplication is a powerful driving force of neo- and subfunctionalization during plant evolution [54]. The four SPA genes of Arabidopsis are indeed not fully redundant but have partially distinct functions during Arabidopsis development $[13,15]$. At least some of the functional divergence, the one between Arabidopsis SPA1 and SPA2, has been mapped to the respective SPA protein sequence rather than the promoter sequences [27]. Hence, evidence strongly suggests that the four Arabidopsis SPA proteins are not identical in function but provide some degree of specificity to the COP1/SPA E3 ligase activity. The failure of PpSPAb and OsSPA1 to fully replace AtSPA1 in Arabidopsis supports that such functional divergence has occurred in the course of land plant evolution. While this is very reasonable, it is nevertheless significant that COP1 coding sequences did not functionally co-diverge with $S P A$ sequences, especially considering that both proteins carry very similar WD40-repeat domains in their C-termini which both are able to bind and thereby recognize the same substrate proteins [11]. Hence, COP1 must provide a core function to the COP1/SPA complex that hinders evolutionary divergence, and this core function is likely modified by divergent SPA proteins. 
OsSPA1 was capable of restoring a wild-type phenotype in dark-grown spa triple mutant seedlings - though at low efficiency - but not in light-grown seedlings or adult plants. We consider two possible scenarios to explain this dark-specific complementation by OsSPA1: OsSPA1 activity may be reduced when compared to AtSPA1 and therefore be solely sufficient to restore SPA function in darkness but not in the light. This would be consistent with previous observations showing that mutations in a single SPA gene caused a mutant phenotype only in the light but not in darkness [24,55]. Hence, full SPA activity is much more critical in light-grown seedlings than in dark-grown seedlings, probably because the light-induced inactivation of the COP1/SPA complex causes additional stress on the activity of the complex. Alternatively, OsSPA1 may be hyperinactivated by Arabidopsis photoreceptors and, therefore, lack any activity in the light. This behavior is found in the Arabidopsis SPA2 protein which also shows high activity primarily in dark-grown seedlings [27]. Though the OsSPA1 sequence is more similar to Arabidopsis SPA1 than to SPA2, we do not exclude this possibility. Knocking out the OsSPA1 gene in rice would allow to distinguish between these two possibilities.

The degree of complementation by COP1 orthologs varied with respect to the different aspects of the cop1 mutant phenotype. Most evidently, expression of OsCOP1 or PpCOP1a in light-grown cop1-5 seedlings caused a reduced response to $B$ and FR, a phenotype that is reminiscent of AtCOP1 overexpression rather than of reduced COP1 activity. This observation suggests that OsCOP1 and PpCOP1a maintain higher activity in the light than AtCOP1 and are therefore incompletely inactivated by Arabidopsis photoreceptors when compared to the native Arabidopsis COP1 protein. Hence, photoreceptor and COP1 sequences appear to have co-evolved to allow optimal adaptation of seedling growth to the ambient light environment. When analyzing adult growth and development, PpCOP1a and OsCOP1 fully complemented the cop1-5 leaf expansion phenotype while they only partially complemented the early-flowering phenotype of cop1-5. Since these phenotypes are mediated by distinct substrates, it is evident that the COP1-like proteins from rice and Physcomitrella do not polyubiquitinate all substrates of Arabidopsis COP1 equally well. Hence, functional conservation of COP1 may have varied with respect to the different substrates of COP1. Orthologs of known COP1/SPA substrates exist in $P$. patens, such as two PpHY5 and three PpCO-like (PpCOL) proteins [56-58]. A role of PpHY5 in moss light responses was described [58]. Hence, if PpCOPla acts as a light-regulated ubiquitin ligase in mosses as well, it may indeed mediate degradation of the PpHY5 protein. In the future, it will be interesting to elucidate whether there is a COP1/SPA E3 ligase in Physcomitrella and, if so, which substrates are recognized.

\section{Conclusions}

Our results show that COP1 protein sequences from Physcomitrella, rice and Arabidopsis are functionally conserved, while the sequences of the SPA proteins showed considerable functional divergence. This may - at least in part - reflect the fact that COP1 is a single copy gene in flowering plants, while SPA proteins are encoded by a small gene family of two to four members, thus possibly allowing sub- or neofunctionalization. Light responses are very distinct in mosses and angiosperms [39]. Whether these differences reflect distinct signaling pathways including the recruitment of different transcription factors into the light signaling network needs to be resolved.

\section{Methods}

\section{Sequences and ortholog prediction}

Arabidopsis protein sequences correspond to the loci SPA1 (At2g46340.1), SPA2 (At4g11110.1), SPA3 (At3g15354.1), SPA4 (At1g53090.1) and COP1 (At2g32950.1) of the annotated Arabidopsis Col genome TAIR10 annotation [59]. Rice proteins correspond to the loci OsCOP1 (Os02g53140.1), OsSPA1 (Os05g49590.1) and OsSPA3/4 (Os01g52640.3) of the Rice Genome Annotation Project Release 7 [60]. However, based on an amino acid sequence alignment with all other SPAs from Arabidopsis, Physcomitrella and rice, the corresponding reference sequence of OsSPA3/4 (Os01g52640.3) lacks a part of the WD40 repeat domain. Here we used an alternatively spliced sequence, Os01g524630.1, from Genome Annotation Project Release 5 which contains additional WD40 repeats and in our opinion reflects the full-length OsSPA3/4 protein. Physcomitrella proteins correspond to the loci indicated in Figure 1. They are derived from the cosmoss.org Physcomitrella patens V1.6 genome annotation [48].

To conduct a phylogentic reconstruction of the COP1/ SPA genes in Arabidopsis, rice and Physcomitrella, first an all-against-all blast search was performed. To find homologous sequences between these species, blastp + version 2.2.9 [61] was used to build a blast database with protein sequences as indicated in Additional file 7: Table S1 and a blastp search was performed with an e-value cutoff of 10 by using the BLOSUM62 matrix. The resulting blastp results were then filtered by applying a changed version of formula (2) as indicated by [62]. These filtered blastp results were then used with proteinortho version 4.26 [63] to detect co-orthologs within and between these species by using the following options $[-\mathrm{e}=0.01 ;-\mathrm{id}=11 ;-\operatorname{cov}=$ $0.25 ; \quad-$ conn $=0.1 ; \quad-\mathrm{m}=0.75 ; \quad$ - pairs; $\quad-$ selfblast $; \quad$-blastdone]. The proteinortho results were filtered for COP1 (AT2G32950.1), SPA1 (AT2G46340.1), SPA2 (AT4G11110.1), SPA3 (AT3G15354.1), SPA4 (AT1G53090.1), RUP1 (AT5G 52250.1), RUP2 (AT5G23730.1) and all resulting co-orthologs were used for further analysis and were screened for protein domains by InterProScan version 5 [64]. The program ncoils 
(based on [65]) is used by InterProScan with default settings to predict coiled-coils domains. In addition to standard settings we used different sliding window parameters $[14,21,28]$ for the coiled-coils domain predictions which are highlighted in Additional file 3: Figure S3. For the phylogenetic reconstruction RUP1 and RUP2 were chosen as an outgroup gene family since both also contain WD40 repeats like the COP1/SPA genes but lack functional domains further upstream. These genes could be used to root COP1/SPA phylogenetic trees.

\section{Phylogenetic analysis}

A multiple sequence alignment (MSA) was calculated with MAFFT L-INS-i version 7.037b [66], ProbCons version 1.12 [67], Muscle version 3.8.31 [68] and T-coffee version 8.99 [69] with default settings and subsequently combined into an optimal alignment using the combiner function of T-coffee. The MSA was visualized and manually curated using Jalview version 2.8 [70] (Additional file 2: Figure S2, Additional file 4: Figure S4, Additional file 5: Figure S5). The JTT + G + I + F model was selected as the best fitting amino acid substitution model according to the Bayesian Information Criterion in ProtTest version 3.3 [71]. To reconstruct the phylogeny we used MrBayes 3.2.2 [72] and RAxML version 8.0.2 [73].

For MrBayes we initiated two runs of four Markovchain Monte Carlo (MCMC) chains of $2 \times 10^{7}$ generations each from a random starting tree, sampling every 1,000 generations [additional settings: rates = invgamma, ngammacat $=4$, aamodelpr $=$ JTT]. A 25\% burn-in was chosen and convergence was assessed by standard deviation of split frequencies falling below 0.005 .

RAxML conducted 1,000 non-parametric bootstrap inferences with the rapid hill-climbing mode using the PROTGAMMAIJTTF model [additional settings: -d -b -\#1000]. The bootstrap replicates were used to build a consensus tree applying the majority rule option (-m PROTGAMMAIJTTF -J MR). Phylogenetic trees were rooted by the RUP outgroup gene family and visualized with Figtree version 1.4.0 (http://tree.bio.ed.ac.uk/software/figtree/).

\section{Plant material, light sources and growth conditions}

All mutant genotypes used were described previously: cop1-4 (Col-0), cop1-5 (Ws) [51], spa1-7 spa3-1 spa4-1 and spa1-7 spa2-1 spa3-1 (both Col) [34]. Light sources, seedling growth conditions and determination of seedling and adult traits were described previously [27].

\section{Plasmid constructions, plant transformations and selection of transgenic plants}

All ORF clones were designed based on the sequence information provided in the databases described above. To generate COP1 expression clones, AtCOP1, OsCOP1 and $P p C O P 1 a$ ORFs were amplified using gene-specific primers with attached attB sites and the amplified sequences were subsequently cloned into the pDONR221 entry vector by Gateway cloning according to the manufacturer's instructions. AtCOP1 and PpCOP1a ORFs were amplified from cDNA derived from Arabidopsis seedlings or Physcomitrella gametophores, respectively. OsCOP1 was amplified from a full-length cDNA clone obtained from National Institute for Agrobiological Sciences (NIAS), Japan. The obtained Entry clones were recombined with the pGJ2169 GW binary destination vector (kindly provided by George Coupland) containing the $35 \mathrm{~S}$ promoter before the Gateway cassette. The final destination vectors were transformed into homozygous cop1-4 and heterozygous cop1-5/+ mutants. Transgenic plants were selected on Basta herbicide. In the cop1-4 background, at least 25 independent transgenic lines per construct were analyzed in the T1 (flowering time, leaf size) and T2 (seedling deetiolation) generations. In the cop1-5 background, lines homozygous for cop1-5 and the respective transgene were generated by selecting for kanamycin resistance (cop1-5), the absence of the native COP1 transcript and the presence of the introduced transgene (Basta resistance).

SPA expression clones were constructed as follows: First, 2260 bp or 1309 bp of the Arabidopsis SPA1 or $S P A 45^{\prime}$ regulatory regions preceding the ATG start codon, respectively ( $p S P A 1, p S P A 4)$, were amplified from previously constructed plasmids using primers containing HindIII or SdaI restriction sites, respectively, and subsequently cloned into unique HindIII or SdaI restriction sites, respectively, of the pGWB1 destination vector [74]. These modified pGWB1 destination vectors now have Gateway cassettes after the $p S P A 1$ or $p S P A 4$ promoters, respectively. Second, Entry clones carrying the ORFs of SPA sequences were generated after amplifying the ORF of AtSPA1 and AtSPA4 from Arabidopsis cDNA, the OsSPA1 ORF from a full-length cDNA clone obtained from NIAS, Japan, and the Physcomitrella $S P A b$ ORF from cDNA synthesized from Physcomitrella gametophores (for all primer sequences, see Additional file 8: Table S2). Third, the modified pGWB1 destination vectors described above were recombined with the Entry clones containing the ORFs from AtSPA1, OsSPA1, PpSPAb and AtSPA4, respectively, using Gateway LR technology to generate pSPA1::AtSPA1/ OsSPA1/PpSPAb vectors and $p A t S P A 4:: A t S P A 4 / P p S P A b$ vectors. These binary vectors were transformed into Arabidopsis spa 1 spa 3 spa 4 and spa 1 spa 2 spa 3 mutants.

\section{RNA isolation and transcript analysis}

RNA was isolated and reverse-transcribed as described previously [27]. SPA ORFs were amplified by semi-quantitative RT-PCR using gene-specific primers (Additional file 8: Table S2). PCR products were resolved by agarose electrophoresis and subsequent staining with ethidium bromide. 


\section{Availability of supporting data}

The data sets supporting the results of this article are included within the article and its additional files.

\section{Additional files}

Additional file 1: Figure S1. Phylogeny and domain structure of COP1 and SPA gene family in Arabidopsis, rice and Physcomitrella. A. Phylogenetic tree based on Bayesian inference created with COP1 and SPA homologs in three plant species. The Bayesian consensus phylogeny was constructed on a manual curated multiple sequence alignment rooted by the RUP gene family as an outgroup. Numbers on internal branches indicate Bayesian posterior probabilities. Line thickness corresponds to posterior probabilities. Detailed settings used for tree construction and tree plotting can be obtained from the methods chapter. B. Phylogenetic tree based on maximum likelihood created with COP1 and SPA homologs in three plant species. Consensus tree build by the majority rule of bootstrap replicates. Numbers on internal branches indicate support values of bootstrap in percent. Line corresponds to bootstrap support values. Detailed settings used for tree construction and tree plotting can be obtained from the methods chapter. C. Protein domains important for COP1 and SPA gene function obtained by InterProScan5. For each protein the domain structures obtained by InterProScan5 were plotted next to each protein. Individual domain position corresponds to their absolute position along the analyzed protein; red boxes, IPR013083 - Zinc finger, RING/FYVE/PHD - type; orange boxes, IPR001841 - Zinc finger, RING - type; light green boxes, IPR011009 - Protein kinase - like domain; green boxes, IPR000719 Protein kinase domain; blue boxes, IPR015943/IPR017986 - WD40/YVTN repeat - like - containing domain; light blue boxes represent number of WD40 repeats, SM00320 - WD40 repeat; grey boxes represent number of coiled-coil occurrence based on Coils prediction.

Additional file 2: Figure S2. Multiple sequence alignment of Arabidopsis, rice and Physcomitrella COP1 protein sequences. Sequence alignment displayed using Jalview version 2.8. Protein stretches belonging to InterProScan5 domain IPR001841 - Zinc finger, RING - type are highlighted in orange; predicted occurrence of coiled-coil domains are highlighted in grey; WD40 repeats, SM00320 - WD40 repeat are highlighted in light blue.

Additional file 3: Figure S3. Prediction of coiled-coil domains in Arabidopsis, rice and Physcomitrella COP1 and SPA protein sequences. Prediction of coiled-coil domains were obtained from COILS (version 2.2) with three different sliding window parameters and the MTIDK matrix. Results indicating prediction probabilities for each window were plotted alongside the protein length. Next to each protein name obtained by the used sequence databases an alias was attached.

Additional file 4: Figure S4. Multiple sequence alignment of Arabidopsis, rice and Physcomitrella SPA-related protein sequences. Sequence alignment displayed using Jalview version 2.8. Protein stretches belonging to InterProScan5 domain IPR011009 - Protein kinase - like domain are highlighted in light green; IPR000719 - Protein kinase domain are highlighted in green; predicted occurrence of coiled-coil domains are highlighted in grey; WD40 repeats, SM00320 - WD40 repeat are highlighted in light blue.

Additional file 5: Figure S5. Multiple sequence alignment of Arabidopsis, rice and Physcomitrella RUP1-related protein sequences. Sequence alignment displayed using Jalview version 2.8. Protein stretches representing WD40 repeats, SM00320 - WD40 repeat are highlighted in light blue.

Additional file 6: Figure S6. Hypocotyl elongation response of wild-type and transgenic cop1-5 mutant seedlings to Rc (A) and FRc (B) Transgenic seedlings express AtCOP1, OsCOP1 or PpCOP1 under the control of the 355 promoter. Two to three independent transgenic lines are shown. cop 1-5 mutant seeds failed to germinate due to the seedling-lethal phenotype and are therefore not shown. Error bars indicate the standard error of the mean (SEM).

Additional file 7: Table S1. List of sequence databases used.

Additional file 8: Table S2. Primer sequences.

\section{Competing interests}

The authors declare no competing interests.

\section{Authors' contributions}

AR, SD and UH carried out the molecular and genetic studies. KU and SR performed the sequence and phylogenetic analyses. All authors drafted, read and approved the final manuscript.

\section{Acknowledgements}

We are grateful to the National Institute for Agrobiological Sciences (NIAS) in Japan for providing full-length OsCOP1 and OsSPA1 CDNA clones and to George Coupland for the gift of the pGJ2169 GW vector. We thank Sebastian Meller for excellent help in identifying transgenic cop 1-5 mutant lines and Klaus Menrath and the greenhouse staff for expert care of our plants. This work was funded by the Deutsche Forschungsgemeinschaft (SFB635) to U.H. Aashish Ranjan was a recipient of a Ph.D. fellowship from the International Graduate School of Genetics and Functional Genomics from the state Northrhine-Westphalia, Germany. Stephen Dickopf was supported by a Ph.D. fellowship from the International Max-Planck Research School and the University of Cologne.

\section{Author details}

'Botanical Institute and Cluster of Excellence on Plant Sciences (CEPLAS), Biocenter, University of Cologne, Zülpicher Str. 47b, 50674 Cologne, Germany. ${ }^{2}$ Plant Cell Biology, Faculty of Biology, University of Marburg, Karl-von-Frisch-Str. 8, 35043 Marburg, Germany. ${ }^{3}$ Present addresss: Life Sciences Addition \#2237, Section of Plant Biology, UC Davis, One Shields Ave, Davis, CA 95616, USA.

Received: 24 April 2014 Accepted: 24 June 2014 Published: 1 July 2014

\section{References}

1. Kami C, Lorrain S, Hornitschek P, Fankhauser C: Light-regulated plant growth and development. Curr Top Dev Biol 2010, 91:29-66.

2. Whitelam GC, Halliday KJ: Light and plant development. Blackwell 2007, 30:

3. Demarsy E, Fankhauser C: Higher plants use LOV to perceive blue light. Curr Opin Plant Biol 2009, 12(1):69-74

4. Nagatani A: Phytochrome: structural basis for its functions. Curr Opin Plant Biol 2010, 13(5):565-570.

5. Chaves I, Pokorny R, Byrdin M, Hoang N, Ritz T, Brettel K, Essen LO, van der Horst GT, Batschauer A, Ahmad M: The cryptochromes: blue light photoreceptors in plants and animals. Annu Rev Plant Biol 2011, 62:335-364.

6. Heijde M, Ulm R: UV-B photoreceptor-mediated signalling in plants. Trends Plant Sci 2012, 17(4):230-237.

7. Maier A, Schrader A, Kokkelink L, Falke C, Welter B, Iniesto E, Rubio V, Uhrig JF, Hulskamp M, Hoecker U: Light and the E3 ubiquitin ligase COP1/SPA control the protein stability of the MYB transcription factors PAP1 and PAP2 involved in anthocyanin accumulation in Arabidopsis. Plant J 2013, 74(4):638-651

8. Weidler G, Zur Oven-Krockhaus S, Heunemann M, Orth C, Schleifenbaum F, Harter K, Hoecker U, Batschauer A: Degradation of Arabidopsis CRY2 is regulated by SPA proteins and phytochrome A. Plant Cell 2012, 24(6):2610-2623.

9. Li YY, Mao K, Zhao C, Zhao XY, Zhang HL, Shu HR, Hao YJ: MdCOP1 Ubiquitin E3 Ligases Interact with MdMYB1 to Regulate Light-Induced Anthocyanin Biosynthesis and Red Fruit Coloration in Apple. Plant Physiol 2012, 160(2):1011-1022.

10. Hoecker U: Regulated proteolysis in light signaling. Curr Opin Plant Biol 2005, 8(5):469-476.

11. Lau OS, Deng XW: The photomorphogenic repressors COP1 and DET1: 20 years later. Trends Plant Sci 2012, 17(10):584-593.

12. Deng X-W, Caspar T, Quail PH: cop1: A regulatory locus involved in light-controlled development and gene expression in Arabidopsis. Genes Dev 1991, 5:1172-1182.

13. Laubinger $S$, Fittinghoff $K$, Hoecker U: The SPA quartet: a family of WD-repeat proteins with a central role in suppression of photomorphogenesis in Arabidopsis. Plant Cell 2004, 16(9):2293-2306.

14. Rolauffs S, Fackendahl P, Sahm J, Fiene G, Hoecker U: Arabidopsis COP1 and SPA genes are essential for plant elongation but not for acceleration of flowering time in response to a low red light to far-red light ratio. Plant Physiol 2012, 160(4):2015-2027. 
15. Laubinger S, Marchal V, Gentilhomme J, Wenkel S, Adrian J, Jang S, Kulajta C, Braun H, Coupland G, Hoecker U: Arabidopsis SPA proteins regulate photoperiodic flowering and interact with the floral inducer CONSTANS to regulate its stability. Development 2006, 133(16):3213-3222.

16. Jang S, Marchal V, Panigrahi KC, Wenkel S, Soppe W, Deng XW, Valverde F, Coupland G: Arabidopsis COP1 shapes the temporal pattern of CO accumulation conferring a photoperiodic flowering response. Embo $\mathrm{J}$ 2008, 27(8):1277-1288

17. Liu LJ, Zhang YC, Li QH, Sang Y, Mao J, Lian HL, Wang L, Yang HQ: COP1-mediated ubiquitination of CONSTANS is implicated in cryptochrome regulation of flowering in Arabidopsis. Plant Cell 2008, 20(2):292-306.

18. Crocco CD, Holm M, Yanovsky MJ, Botto JF: AtBBX21 and COP1 genetically interact in the regulation of shade avoidance. Plant J 2010, 64(4):551-562.

19. Baumgardt RL, Oliverio KA, Casal JJ, Hoecker U: SPA1, a component of phytochrome A signal transduction, regulates the light signaling current. Planta 2002, 215(5):745-753.

20. Tilbrook K, Arongaus AB, Binkert M, Heijde M, Yin R, Ulm R: The UVR8 UV-B Photoreceptor: Perception. Signaling and Response. Arabidopsis Book 2013, 11:e0164.

21. Zhu D, Maier A, Lee JH, Laubinger S, Saijo Y, Wang H, Qu LJ, Hoecker U, Deng XW: Biochemical characterization of Arabidopsis complexes containing CONSTITUTIVELY PHOTOMORPHOGENIC1 and SUPPRESSOR OF PHYA proteins in light control of plant development. Plant Cell 2008, 20(9):2307-2323.

22. Hoecker $U$, Quail PH: The phytochrome A-specific signaling intermediate SPA1 interacts directly with COP1, a constitutive repressor of light signaling in Arabidopsis. J Biol Chem 2001, 276:38173-38178.

23. Saijo Y, Sullivan JA, Wang H, Yang J, Shen Y, Rubio V, Ma L, Hoecker U, Deng XW: The COP1-SPA1 interaction defines a critical step in phytochrome A-mediated regulation of HY5 activity. Genes Dev 2003, 17(21):2642-2647.

24. Laubinger S, Hoecker U: The SPA1-like proteins SPA3 and SPA4 repress photomorphogenesis in the light. Plant J 2003, 35(3):373-385.

25. Deng XW, Matsui M, Wei N, Wagner D, Chu AM, Feldmann KA, Quail PH: COP1, an Arabidopsis regulatory gene, encodes a protein with both a zinc-binding motif and a G beta homologous domain. Cell 1992, 71(5):791-801.

26. Hoecker U, Tepperman JM, Quail PH: SPA1, a WD-repeat protein specific to phytochrome A signal transduction. Science 1999, 284:496-499.

27. Balcerowicz M, Fittinghoff K, Wirthmueller L, Maier A, Fackendahl $P$, Fiene G, Koncz C, Hoecker U: Light exposure of Arabidopsis seedlings causes rapid de-stabilization as well as selective post-translational inactivation of the repressor of photomorphogenesis SPA2. Plant J 2011, 65(5):712-723.

28. Pacin $M$, Legris $M$, Casal JJ: COP1 re-accumulates in the nucleus under shade. Plant J 2013, 75(4):631-641.

29. Von Arnim AG, Deng X-W: Light inactivation of arabidopsis photomorphogenic repressor COP1 involves a cell-specific regulation of its nucleocytoplasmic partitioning. Cell 1994, 79:1035-1045.

30. Lian HL, He SB, Zhang YC, Zhu DM, Zhang JY, Jia KP, Sun SX, Li L, Yang HQ: Blue-light-dependent interaction of cryptochrome 1 with SPA1 defines a dynamic signaling mechanism. Genes Dev 2011, 25(10):1023-1028.

31. Liu B, Zuo Z, Liu H, Liu X, Lin C: Arabidopsis cryptochrome 1 interacts with SPA1 to suppress COP1 activity in response to blue light. Genes Dev 2011, 25(10):1029-1034.

32. Zuo Z, Liu H, Liu B, Liu X, Lin C: Blue light-dependent interaction of CRY2 with SPA1 regulates COP1 activity and floral initiation in Arabidopsis. Curr Biol 2011, 21(10):841-847.

33. Pacin M, Legris M, Casal JJ: Rapid decline in nuclear costitutive photomorphogenesis 1 abundance anticipates the stabilization of its target elongated hypocotyl5 in the light. Plant Physio/ 2014 164(3):1134-1138

34. Fittinghoff $K$, Laubinger S, Nixdorf M, Fackendahl P, Baumgardt RL, Batschauer A, Hoecker U: Functional and expression analysis of Arabidopsis SPA genes during seedling photomorphogenesis and adult growth. Plant J 2006, 47(4):577-590.

35. Tanaka N, Itoh H, Sentoku N, Kojima M, Sakakibara H, Izawa T, Itoh J, Nagato Y: The COP1 ortholog PPS regulates the juvenile-adult and vegetative-reproductive phase changes in rice. Plant Cell 2011, 23(6):2143-2154.
36. Sullivan JA, Gray JC: The pea light-independent photomorphogenesis1 mutant results from partial duplication of COP1 generating an internal promoter and producing two distinct transcripts. Plant Cell 2000, 12(10):1927-1938

37. Weller JL, Hecht V, Vander Schoor JK, Davidson SE, Ross JJ: Light regulation of gibberellin biosynthesis in pea is mediated through the COP1/HY5 pathway. Plant Cell 2009, 21(3):800-813.

38. Marine JC: Spotlight on the role of COP1 in tumorigenesis. Nat Rev Cancer 2012, 12(7):455-464

39. Lamparter T: Photomorphogenesis of mosses. 3rd edition. Dordrecht, The Netherlands: Springer; 2006

40. Wolf L, Rizzini L, Stracke R, Ulm R, Rensing SA: The molecular and physiological responses of Physcomitrella patens to ultraviolet-B radiation. Plant Physiol 2010, 153(3):1123-1134.

41. Richardt S, Lang D, Reski R, Frank W, Rensing SA: PlanTAPDB, a phylogeny-based resource of plant transcription-associated proteins. Plant Physio/ 2007, 143(4):1452-1466.

42. Rensing SA, Lang D, Zimmer AD, Terry A, Salamov A, Shapiro H, Nishiyama T, Perroud PF, Lindquist EA, Kamisugi Y, Tanahashi T, Sakakibara K, Fujita T, Oishi K Shin-I T, Kuroki Y, Toyoda A, Suzuki Y, Hashimoto S, Yamaguchi K, Sugano S, Kohara Y, Fujiyama A, Anterola A, Aoki S, Ashton N, Barbazuk WB, Barker E, Bennetzen $J$, Blankenship R: The Physcomitrella genome reveals evolutionary insights into the conquest of land by plants. Science 2008, 319(5859):64-69.

43. International rice genome sequencing project: The map-based sequence of the rice genome. Nature 2005, 436(7052):793-800.

44. Possart A, Hiltbrunner A: An evolutionarily conserved signaling mechanism mediates far-red light responses in land plants. Plant Cell 2013, 25(1):102-114

45. Chen YR, Su YS, Tu SL: Distinct phytochrome actions in nonvascular plants revealed by targeted inactivation of phytobilin biosynthesis. Proc Natl Acad Sci U S A 2012, 109(21):8310-8315.

46. Jaedicke K, Lichtenthaler AL, Meyberg R, Zeidler M, Hughes J: A phytochrome-phototropin light signaling complex at the plasma membrane. Proc Natl Acad Sci U S A 2012, 109(30):12231-12236.

47. Riese M, Zobell O, Saedler H, Huijser P: SBP-domain transcription factors as possible effectors of cryptochrome-mediated blue light signalling in the moss Physcomitrella patens. Planta 2008, 227(2):505-515.

48. Zimmer AD, Lang D, Buchta K, Rombauts S, Nishiyama T, Hasebe M, Van de Peer $Y$, Rensing SA, Reski R: Reannotation and extended community resources for the genome of the non-seed plant Physcomitrella patens provide insights into the evolution of plant gene structures and functions. BMC Genomics 2013, 14:498.

49. Gruber H, Heijde M, Heller W, Albert A, Seidlitz HK, Ulm R: Negative feedback regulation of UV-B-induced photomorphogenesis and stress acclimation in Arabidopsis. Proc Natl Acad Sci U S A 2010, 107(46):20132-20137.

50. Heijde M, Ulm R: Reversion of the Arabidopsis UV-B photoreceptor UVR8 to the homodimeric ground state. Proc Natl Acad Sci U S A 2013, 110(3):1113-1118.

51. McNellis TW, Von Arnim AG, Araki T, Komeda Y, Miséra S, Deng X-W: Genetic and molecular analysis of an allelic series of cop 1 mutants suggests functional roles for the multiple protein domains. Plant Cell 1994, 6:487-500.

52. Ranjan A, Fiene G, Fackendahl P, Hoecker U: The Arabidopsis repressor of light signaling SPA1 acts in the phloem to regulate seedling de-etiolation, leaf expansion and flowering time. Development 2011, 138(9):1851-1862.

53. Yang J, Wang $\mathrm{H}$ : The central coiled-coil domain and carboxyl-terminal WD-repeat domain of Arabidopsis SPA1 are responsible for mediating repression of light signaling. Plant J 2006, 47(4):564-576.

54. Rensing SA: Gene duplication as a driver of plant morphogenetic evolution. Curr Opin Plant Biol 2014, 17C:43-48.

55. Hoecker $U, X u Y$, Quail PH: SPA1: A new genetic locus involved in phytochrome A-specific signal transduction. Plant Cell 1998, 10:19-33.

56. Zobell O, Coupland G, Reiss B: The family of CONSTANS-like genes in Physcomitrella patens. Plant Biol (Stuttg) 2005, 7(3):266-275.

57. Shimizu M, Ichikawa K, Aoki S: Photoperiod-regulated expression of the PpCOL1 gene encoding a homolog of CO/COL proteins in the moss Physcomitrella patens. Biochem Bioph Res Co 2004, 324(4):1296-1301.

58. Yamawaki S, Yamashino T, Nakanishi H, Mizuno T: Functional characterization of HY5 homolog genes involved in early light-signaling in Physcomitrella patens. Biosci Biotechnol Biochem 2011, 75(8):1533-1539.

59. Lamesch P, Berardini TZ, Li DH, Swarbreck D, Wilks C, Sasidharan R, Muller R, Dreher K, Alexander DL, Garcia-Hernandez M, Karthikeyan AS, Lee CH, Nelson 
WD, Ploetz L, Singh S, Wensel A, Huala E: The Arabidopsis Information Resource (TAIR): improved gene annotation and new tools. Nucleic Acids Res 2012, 40(D1):D1202-D1210.

60. Kawahara Y, De La Bastide M, Hamilton JP, Kanamori H, McCombie WR, Ouyang S, Schwartz DC, Tanaka T, Wu J, Zhou S, Childs KL, Davidson RM, Lin H, Quesada-Ocampo L, Vaillancourt B, Sakai H, Lee SS, Kim J, Numa H, Itoh T, Buell CR, Matsumoto T: Improvement of the Oryza sativa Nipponbare reference genome using next generation sequence and optical map data. Rice (New York, NY) 2013, 6(1):4.

61. Camacho C, Coulouris G, Avagyan V, Ma N, Papadopoulos J, Bealer K, Madden TL: BLAST+: architecture and applications. BMC Bioinform 2009, 10:421.

62. Rost B: Twilight zone of protein sequence alignments. Protein Eng 1999, 12(2):85-94.

63. Lechner M, Findeiss S, Steiner L, Marz M, Stadler PF, Prohaska SJ: Proteinortho: Detection of (Co-)orthologs in large-scale analysis. Bmc Bioinform 2011, 12:124.

64. Jones P, Binns D, Chang HY, Fraser M, Li W, McAnulla C, McWilliam H, Maslen J, Mitchell A, Nuka G, Pesseat S, Quinn AF, Sangrador-Vegas A, Scheremetjew M, Yong SY, Lopez R, Hunter S: InterProScan 5: genome-scale protein function classification. Bioinform 2014, 30:1236-1240.

65. Lupas A, Vandyke M, Stock J: PREDICTING COILED COILS FROM PROTEIN SEQUENCES. Science 1991, 252(5009):1162-1164.

66. Katoh K, Standley DM: MAFFT Multiple Sequence Alignment Software Version 7: Improvements in Performance and Usability. Mol Biol Evol 2013, 30(4):772-780

67. Do CB, Mahabhashyam MSP, Brudno M, Batzoglou S: ProbCons: Probabilistic consistency-based multiple sequence alignment. Genome Res 2005, 15(2):330-340.

68. Edgar RC: MUSCLE: multiple sequence alignment with high accuracy and high throughput. Nucleic Acids Res 2004, 32(5):1792-1797.

69. Notredame C, Higgins DG, Heringa J: T-Coffee: A novel method for fast and accurate multiple sequence alignment. J Mol Biol 2000, 302(1):205-217.

70. Waterhouse AM, Procter JB, Martin DMA, Clamp M, Barton GJ: Jalview Version 2-a multiple sequence alignment editor and analysis workbench. Bioinform 2009, 25(9):1189-1191.

71. Darriba D, Taboada GL, Doallo R, Posada D: ProtTest 3: fast selection of best-fit models of protein evolution. Bioinform 2011, 27(8):1164-1165.

72. Ronquist F, Huelsenbeck JP: MrBayes 3: Bayesian phylogenetic inference under mixed models. Bioinform 2003, 19(12):1572-1574.

73. Stamatakis A: RAxML version 8: a tool for phylogenetic analysis and post-analysis of large phylogenies. Bioinform 2014, 30(9):1312-1313.

74. Nakagawa T, Kurose T, Hino T, Tanaka K, Kawamukai M, Niwa Y, Toyooka K, Matsuoka K, Jinbo T, Kimura T: Development of series of gateway binary vectors, $\mathrm{pGWBs}$, for realizing efficient construction of fusion genes for plant transformation. J Biosci Bioeng 2007, 104(1):34-41.

doi:10.1186/1471-2229-14-178

Cite this article as: Ranjan et al:: Functional analysis of COP1 and SPA orthologs from Physcomitrella and rice during photomorphogenesis of transgenic Arabidopsis reveals distinct evolutionary conservation. BMC Plant Biology 2014 14:178.

\section{Submit your next manuscript to BioMed Central and take full advantage of:}

- Convenient online submission

- Thorough peer review

- No space constraints or color figure charges

- Immediate publication on acceptance

- Inclusion in PubMed, CAS, Scopus and Google Scholar

- Research which is freely available for redistribution 